\title{
Prevalence and Risk Factors for Preprocedural Medication Errors in Patients With Atrial Fibrillation and Atrial Flutter
}

\author{
Mark K. Tuttle ${ }^{\mathrm{a}, \mathrm{b}} \mathbb{D}^{\mathbb{0}}$, Nicholas Spetko ${ }^{\mathrm{Q}}$, Jonathan Waks ${ }^{\mathrm{a}}$, Kalon K.L. Ho ${ }^{\mathrm{a}}$, \\ Dhruv S. Kazi ${ }^{a}{ }^{\circledR}$, Kunal Tandon ${ }^{\mathrm{a}}{ }^{(0}$, Peter Zimetbaum ${ }^{\mathrm{a}}{ }^{(0)}$
}

\begin{abstract}
Perfect adherence to anticoagulant medications is an important aspect of care for patients with atrial fibrillation undergoing cardiac electrophysiology procedures to minimize the risk of stroke. Despite this, adherence remains imperfect as is associated with added cost of additional procedures (e.g., transesophageal echocardiography) and administrative burden. We sought to identify characteristics of such patients and predictors of medication errors at Beth Israel Deaconess Medical Center.
\end{abstract}

Keywords: Atrial fibrillation; Medication adherence; Medication errors

\section{Introduction}

The use of anticoagulants in patients with atrial fibrillation (AF) reduces the burden of thromboembolic complications, but adherence in real world populations is suboptimal. The increasing use of direct-acting oral anticoagulants (DOACs), which do not require regular laboratory-based monitoring, has only marginally improved rates of adherence over warfarin, with rates of adherence with DOACs as low as $50 \%$ in some studies $[1,2]$.

Certain cardiac electrophysiology procedures, including direct current cardioversion (DCCV), pulmonary vein isolation (PVI), and atrial flutter ablation (AFA), are associated with an increased thromboembolic risk, which is substantially reduced by ensuring perfect adherence to anticoagulation therapy in the 3 weeks preceding the procedure. Failure to so has been associated with a $6.8 \%$ rate of systemic embolism after cardioversion compared with $0.8 \%$ when there is adequate anticoagulation $(\mathrm{P}=0.012)$ [3].

Because of this risk, non-adherence to anticoagulation pri-

Manuscript submitted April 19, 2021, accepted May 7, 2021

Published online July 9, 2021

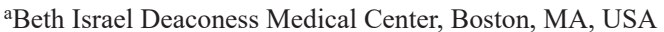

${ }^{b}$ Corresponding Author: Mark K. Tuttle, Beth Israel Deaconess Medical Center, 185 Pilgrim Road, Boston, MA 02215, USA.

Email:mtuttle1@bidmc.harvard.edu or to one of these procedures may necessitate a transesophageal echocardiogram (TEE) to evaluate the possibility of intracardiac thrombus, or the procedure may have to be rescheduled. We sought to understand the rate and predictors of incomplete adherence to anticoagulation prior to procedures for $\mathrm{AF}$ and atrial flutter as well as the clinical administrative burden associated with managing these lapses in adherence.

\section{Materials and Methods}

We conducted a retrospective cohort study of all outpatient DCCVs, PVIs, and AFAs scheduled at Beth Israel Deaconess Medical Center (BIDMC) over a 1-year period from March 29, 2019 until March 28, 2020. We used billing data to collect components of the $\mathrm{CHA}_{2} \mathrm{DS} 2-\mathrm{VASc}$ score [4] using select ICD-10 codes as has been previously described [5]. Patients' ZIP codes were used to determine the median income and poverty rate of the patients' areas of residence using publicly available data from the US Census Bureau [6] since socioeconomic status has been shown to be a risk factor for non-adherence [7]. This project was approved by the BIDMC Institutional Review Board and was in ethical compliance with the Declaration of Helsinki.

We identified all reported medication problems in the 3 weeks preceding a scheduled procedure, consisting of missed or wrong doses of DOACs and subtherapeutic international normalized ratio (INR) if a warfarin was used. Errors were identified by a team of registered nurses who performed an intake interview with each patient on the day prior to their scheduled procedure. We also recorded the action taken by the responsible physician once a medication problem was discovered: procedure cancellation, addition of a TEE, or continuing with the procedure as scheduled. These correspondences took place via an email distribution list which was monitored to determine the volume of emails pertinent to anticoagulation issues.

Statistical analysis was performed using JMP/14 software (SAS Institute Incorporated, Cary, North Carolina). Comparisons were performed with Fisher's exact test for categorical variables and $t$-test for continuous variables.

\section{Results}

A total of 1,248 relevant outpatient electrophysiology proce- 
Table 1. Patient Characteristics

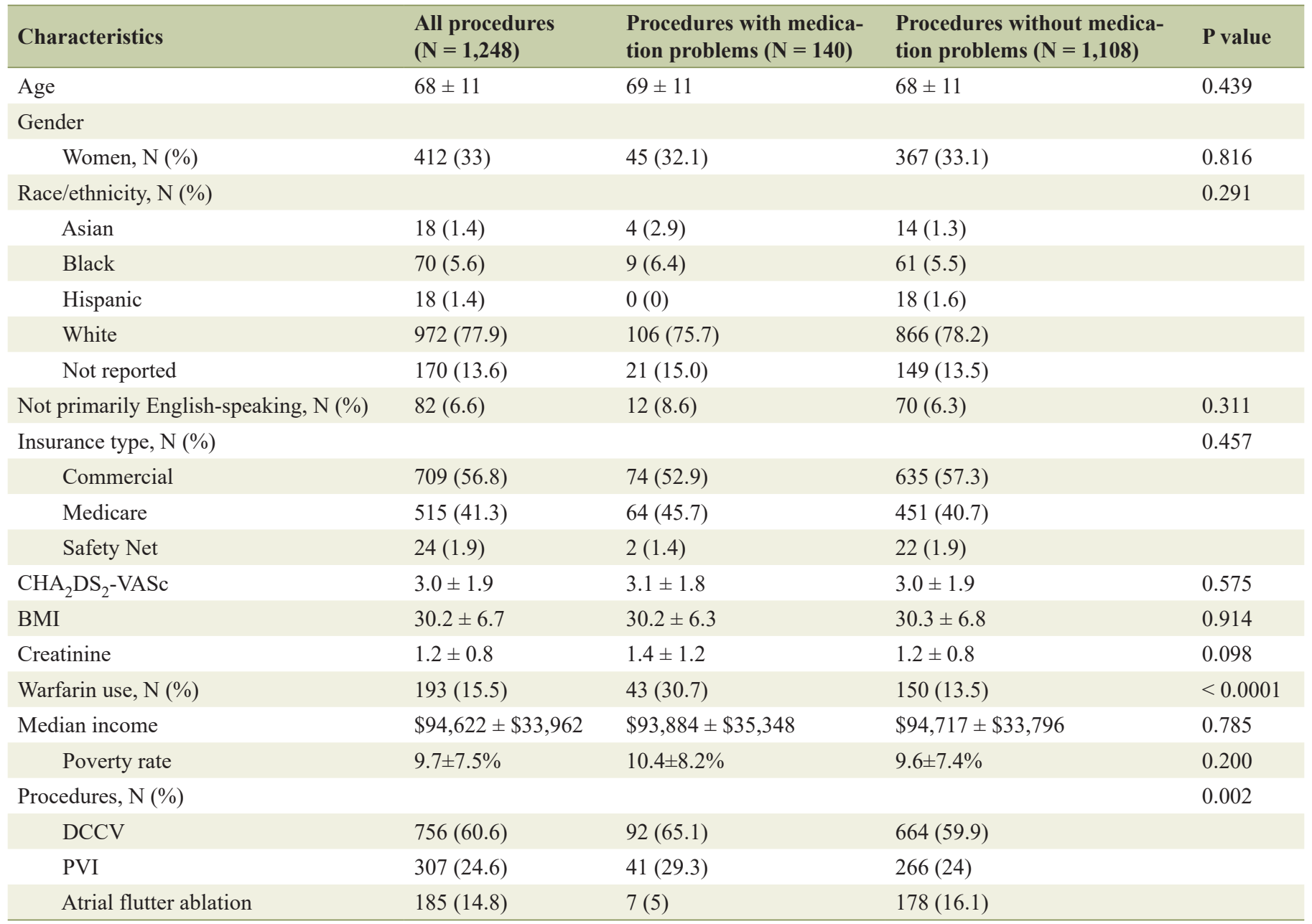

Unless otherwise specified, parameters are expressed as mean \pm standard error. The listed $P$ values relate to the comparison of procedures with medication problems versus procedures without medication problems. BMI: body mass index; $\mathrm{CHA}_{2} \mathrm{DS}_{2}$-VASc: congestive heart failure, hypertension, age, diabetes mellitus, stroke/systemic embolism, vascular disease; DCCV: direct current cardioversion; PVI: pulmonary vein isolation.

dures were scheduled during the study period, consisting of 756 DCCVs, 307 PVIs, and 185 AFAs.

Mean age was 68 years, and 33\% were women. Other patient characteristics are detailed in Table 1. Prescribed anticoagulants included $85.5 \%$ DOACs and $15.5 \%$ warfarin. Among DOACs, rates of use of apixaban, rivaroxaban, and dabigatran were $65.6 \%, 31.9 \%$, and $2.5 \%$, respectively.

Among patients scheduled for these procedures, there were 140 medication problems affecting $11.2 \%$ of procedures. A total of $31 \%$ occurred in patients on warfarin and the remainder in patients receiving DOACs. There was no difference in age, body mass index, $\mathrm{CHA}_{2} \mathrm{DS}_{2}$-VASc score, serum creatinine, insurance type, race, or English-speaking status between the two groups. Median income and poverty rate were not different across groups in the residential ZIP codes of the two groups.

Specific medication problems included missed dose $(\mathrm{N}=$ $85,60.7 \%)$, incorrect dose $(\mathrm{N}=12,8.6 \%)$, and subtherapeutic INR $(\mathrm{N}=34,30.7 \%)$ (Fig. 1$)$. As a result, 100 patients $(71.4 \%)$ had an add-on TEE and 22 patients (15.7\%) had the scheduled procedure cancelled (Fig. 2). Despite the medication problem, 18 patients $(12.8 \%)$ were able to have their procedure as planned (for instance, due to a nearly in-range INR or perception of low embolic risk). Among the 100 patients who underwent TEE add-on, four (4\%) had intracardiac thrombi detected.

There was no difference in the rate of errors among patients taking once-daily DOACS versus twice daily DOACs $(\mathrm{P}=0.10)$.

During the study period, there were an average of 3.1 emails per workday ( 816 per year), or 0.7 emails per procedure $(1,248$ procedures) regarding care coordination for medication problems.

Direct costs of add-on TEEs, which would not have otherwise been required in the absence of medication problems, resulted in nearly a half-million dollars in additional charges to payors. There were also indirect costs of administrative care coordination (e.g., email) which remain challenging to quantify. 


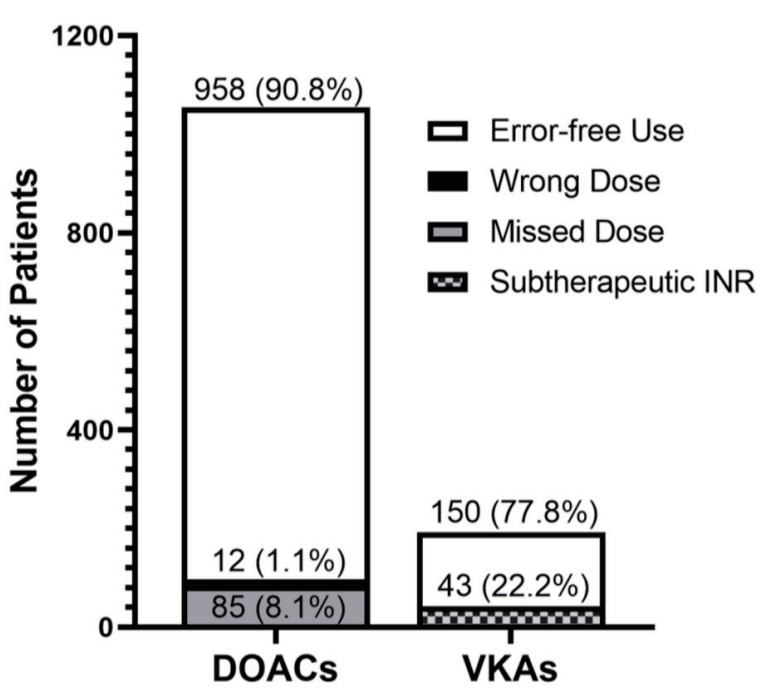

Figure 1. Failure mode of anticoagulation strategy. Types, numbers, and frequencies of medication problems among patients prescribed DOACs and VKAs. DOACs: direct-acting oral anticoagulants; VKAs: vitamin $\mathrm{K}$ antagonists.

\section{Discussion}

Among patients scheduled to undergo 1,248 procedures at a single center, medication errors related to the required anticoagulation therapy in the 3 weeks prior to the procedure occurred in $11.2 \%$ of patients, requiring the addition of TEE in the majority of these patients. Warfarin use was associated with more frequent medication errors than the use of DOACs. Strategies to improve adherence to anticoagulation therapy in this high-risk period are needed.

\section{Limitations}

Unlike with warfarin, confirmatory serum testing for DOACs is not routinely available or utilized, possibly leading to underreporting of medication errors in patients taking DOACs; patients' self-report of DOAC adherence may be inaccurate.

\section{Acknowledgments}

None to declare.

\section{Financial Disclosure}

None to declare.

\section{Conflict of Interest}

Mark Tuttle, Nicholas Spetko, Kalon Ho, Jon Waks, Dhruv Kazi, Kunal Tandon have no conflict of interest to disclose.

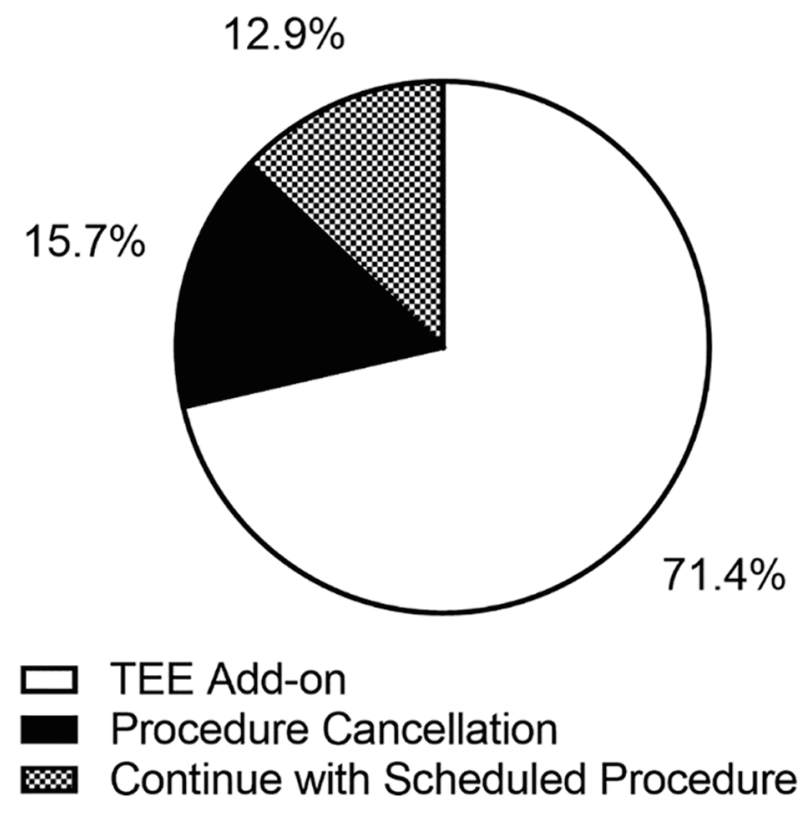

Figure 2. Actions taken in response to medication problems. Frequencies of management strategies were undertaken to address medication problems. TEE: transesophageal echocardiogram.

Peter Zimetbaum: consulting services for Abbott.

\section{Informed Consent}

Not applicable due to retrospective nature of this study.

\section{Author Contributions}

MT and PZ conceived of the project, performed data acquisition, and contributed to writing of the manuscript and data analysis. NS performed data acquisition and contributed to revising of the manuscript. DK and KT helped revise the manuscript. KH and JW contributed to data analysis and helped revise the manuscript.

\section{Data Availability}

The data supporting the findings of this study are available from the corresponding author upon reasonable request.

\section{References}

1. Raparelli V, Proietti M, Cangemi R, Lip GY, Lane DA, Basili S. Adherence to oral anticoagulant therapy in patients with atrial fibrillation. Focus on non-vitamin $\mathrm{K}$ antagonist oral anticoagulants. Thromb Haemost. 2017;117(2):209-218.

2. Boskovic J, Leppee M, Culig J, Eric M. Patient self-reported adherence for the most common chronic medica- 
tion therapy. Scand J Public Health. 2013;41(4):333-335.

3. Bjerkelund CJ, Orning OM. The efficacy of anticoagulant therapy in preventing embolism related to D.C. electrical conversion of atrial fibrillation. Am J Cardiol. 1969;23(2):208-216.

4. Lip GY, Nieuwlaat R, Pisters R, Lane DA, Crijns HJ. Refining clinical risk stratification for predicting stroke and thromboembolism in atrial fibrillation using a novel risk factor-based approach: the euro heart survey on atrial fibrillation. Chest. 2010;137(2):263-272.

5. Tischer T, Schneider R, Lauschke J, Nesselmann C, Klemm A, Diedrich D, Kundt G, et al. Prevalence of atrial fibrilla- tion in patients with high CHADS2- and CHA2DS2VAScscores: anticoagulate or monitor high-risk patients? Pacing Clin Electrophysiol. 2014;37(12):1651-1657.

6. US Census Bureau - Center for Enterprise Dissemination Services and Consumer Innovation [Internet]. [cited May 31, 2020]. Available from: https://data.census.gov/ced$\mathrm{sci} /$.

7. Osborn CY, Kripalani S, Goggins KM, Wallston KA. Financial strain is associated with medication nonadherence and worse self-rated health among cardiovascular patients. J Health Care Poor Underserved. 2017;28(1):499513. 